\title{
The effects of equal and unequal exposures on the Mach-Dvorak stereoillusion
}

\author{
CLAIRE F. MICHAELS and CHARLES STEITZ \\ Lake Forest College, Lake Forest, Illinois 60045 \\ and \\ CLAUDIA CARELLO \\ University of Connecticut, Storrs, Connecticut 06268
}

\begin{abstract}
An onset to onset rule predicts the direction and the magnitude of the displacement in depth that accompanies the successive and equal exposures of a moving object to the two eyes. This rule is ambiguous with respect to identifying the points or regions that are actually "fused"; the disparities of onsets, offsets, and average visual directions are all equal and therefore predict the same illusory displacement. Unequal exposures were used in the present experiment to determine which points or regions do define this disparity. The results revealed that neither onset locations nor offset locations predict the illusion; rather, the fusion of the entire exposed traverses (or their average visual directions) determines the displacement. Because this displacement seems exclusively determined by spatial characteristics, the similarity of this illusion to the Pulfrich effect was questioned.
\end{abstract}

In a previous paper (Michaels, Carello, Shapiro, \& Steitz, 1977), we reported that an onset to onset rule described the binocular integration ${ }^{1}$ of two temporally discrete and dichoptic inputs. This conclusion was drawn from an analysis of the Mach-Dvorak effect in which depth shifts are associated with alternating glimpses of a moving target by the left and right eyes. The present experiment is an extension of that study which attempts to more clearly discern the relationship that is captured by the onset to onset rule.

The onset to onset rule describes the observation that the moving target is displaced in depth to the intersections of the lines connecting each eye with the location of the target at the onset of exposure to that eye (Michaels et al., 1977, Experiment 1). This rule holds for depth displacements both toward and away from the observer (Experiment 2) and up to onset asynchronies of approximately $70 \mathrm{msec}$ (Experiment 3 ). It is important to note that the onset asynchrony per se is not important; it simply describes the displacement of the target given a specific velocity.

There are a number of ways a visual system could deal with the information in the Mach-Dvorak situation that would yield an onset to onset rule. Let us call them "pairing procedures," for they specify the retinal coordinates of the two locales that are "fused." Under conditions of equal exposure to the two eyes, three

The research reported here was supported in part by summer research grants from Lake Forest College to C. Michaels and from the University of Connecticut Research Foundation to C. Carello. Reprint requests should be sent to C. Michaels, Lake Forest College, Lake Forest, Illinois 60045. distinct pairing procedures would yield an onset to onset rule. As can be seen in Figure 1a, the fusion of locations at onset, locations at offset, or the entire visible traverses would predict the same illusory displacement. The purpose of the present experiment was to determine which of these pairing procedures best predicts the observed illusion.

While these various interpretations of the onset to onset rule are not dissociable under typical conditions of Mach-Dvorak stimulation (i.e., with equal exposure durations), they do yield distinct predictions when the

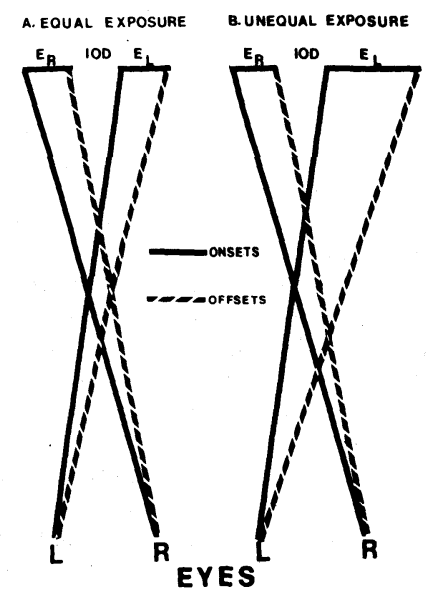

Figure 1. The geometrical configurations for the illusion under conditions of equal exposure duration to the two eyes (Panel A) and under conditions of unequal exposure to the two eyes (Panel B) for one interocular delay (IOD). The intersections of the solid and dotted lines illustrate, respectively, the illusions associated with pairings of onsets and pairings of offsets. 
exposure durations are unequal. Figure $1 \mathrm{~b}$ schematizes the predictions that would be made by the various pairings subsumed by the onset to onset rule. For the particular exposures depicted in Figure 1b, onset-onset pairing would yield a substantially smaller illusion than an offset-offset pairing. The other pairing possibility is that the two entire traverses, one to each eye, are overlaid one upon the other such that the movement is from the onset-onset intersection in Figure $1 \mathrm{~b}$ to the offset-offset intersection. This hypothesis predicts that observers would locate the target somewhere along the diagonal line segment connecting the intersection of onsets and the intersection of offsets.

To summarize, when equal exposures to the two eyes are given in the Mach-Dvorak situation, an onset to onset rule predicts the magnitude of the illusion. Whether the locations at onset are actually paired cannot be discerned from an experiment using equal exposures. Thus, the present experiment sought to determine the precise origin of the onset to onset law by investigating the magnitude of the Mach-Dvorak illusion under conditions of unequal exposure duration.

\section{METHOD}

The illusory displacement of a moving target was measured for two observers under various conditions of exposure duration and onset asynchrony. The two observers, whose corrected vision showed normal scores in a complete screening with a Keystone Telebinocular, aligned a movable marker in the apparent plane of target motion. The target, moving at $50 \mathrm{~cm} / \mathrm{sec}$, was viewed from a distance of $108 \mathrm{~cm}$. For greater detail on the target and adjustable marker, the reader is referred to Michaels et al. (1977).

The temporal manipulations were achieved by having a large $(\mathrm{r}=25 \mathrm{~cm})$ disk rotate in front of the observers' eyes. Two adjustable apertures located at different distances from the center of the disk permitted independent exposure durations to the two eyes at any onset to onset interval. The center of the disk was at eye level to the observer's right. Given the direction of rotation and shape of the apertures, each eye's view was unveiled and occluded bottom to top. To stabilize the eyes horizontally, ${ }^{2}$ observers were required to peer through a narrow slit $(.3 \times 13.5 \mathrm{~cm})$ located $8 \mathrm{~cm}$ from their eyes.

In all, there were 12 conditions. Two of these were control conditions that permitted the measurement of nonillusory localization error; the onsets were simultaneous and the exposures equal. Four conditions with equal exposure durations attempted to reaffirm the onset to onset rule. In these conditions the exposures $(4$ or $8 \mathrm{msec}$ ) were separated by an offsetonset interval of 0 or $4 \mathrm{msec}$, yielding the following conditions: $4_{R}-0-4_{L}, 8_{R}-0-8_{L}, 4_{R}-4-4_{L}$, and $8_{R^{-4-8}}$.

The remaining six conditions employed unequal exposure durations. Two of these directly tested whether locations at onset were "fused." The exposures were simultaneously onset but of unequal duration $\left(4_{R}+8_{L}\right.$ and $\left.8_{R}+4_{L}\right)$. In the final four conditions the unequal exposures were separated by an offset-onset interval of 0 or 4 msec: $4_{R}-0-8_{L}, 8_{R}-0-4_{L}, 4_{R}-4-8_{L}$, and $8_{\mathbf{R}}^{-4-4} \mathbf{L}$. Note that in all conditions with asynchronous onset, exposure to the right eye preceded, in each cycle, exposure to the left eye. Six trials were given in each condition.

The left to right interocular delay ranged from 180 to $200 \mathrm{msec}$, depending on condition, while the onset asynchrony for repeated exposures (cycle time) to the same eye was always 200 msec.

\section{RESULTS AND DISCUSSION}

The depth displacements were averaged over trials within condition. Displacements in the simultaneous and equal exposure conditions showed a relatively consistent localization error on the part of both observers: +2.0 and $+1.4 \mathrm{~cm}$ for the $8 / 8-\mathrm{msec}$ and the 4/4-msec conditions, respectively, for one observer and $+1.5 \mathrm{~cm}$ for both control conditions for the other observer. (Positive displacements are toward the observer.) Because there was neither spatial nor temporal disparity in these two conditions, the scores were considered as a measure of a constant error of localization. As such, the values were subtracted from those observed in the other conditions, leaving a net illusion attributable only to the experimental manipulations and not to localization error (see Michaels et al., 1977, Experiment 2).

In order to compare the observed illusions with the predictions made by the various pairing procedures, the temporal asynchrony associated with each of the corrected illusions was calculated. The computations proceeded as follows: The geometrical model implicit in Figure 1 relates the magnitude of an illusion to a particular interocular spatial disparity. Given the velocity of the target, this spatial disparity can be translated into the interocular temporal disparity associated with that illusion. Let us now compare these disparities with those predicted by the pairing models (on/on, off/off, and total traverse).

The first four rows of Table 1 demonstrate the onset to onset rule; with equal exposure duration to the two eyes, the magnitude of the illusion is that predicted by the amount of onset asynchrony regardless of the relative magnitudes of its components (exposure duration and interocular delay). For example, the illusion is the same for the 8-msec exposure/0-msec delay and the 4-msec exposure/4-msec delay conditions.

However, the onset to onset rule for equal exposures

Table 1

Predicted and Observed Latencies (Milliseconds) by Condition

\begin{tabular}{|c|c|c|c|c|c|c|}
\hline \multicolumn{2}{|c|}{ Exposure } & \multicolumn{5}{|c|}{ Predictions by Pairing } \\
\hline $\mathbf{R}$ & $\mathbf{L}$ & Delay & Ons & Offs & Total & Observed \\
\hline \multicolumn{7}{|c|}{ Equal Exposure Condition } \\
\hline 4 & 4 & 0 & 4 & 4 & 4 & 4.1 \\
\hline 8 & 8 & 0 & 8 & 8 & 8 & 8.0 \\
\hline 4 & 4 & 4 & 8 & 8 & 8 & 8.0 \\
\hline 8 & 8 & 4 & 12 & 12 & 12 & 11.5 \\
\hline \multicolumn{7}{|c|}{ Unequal Exposure (Simultaneous) Condition } \\
\hline 4 & 8 & & 0 & 4 & 2 & 1.7 \\
\hline 8 & 4 & & 0 & -4 & -2 & -2.3 \\
\hline \multicolumn{7}{|c|}{ Unequal Exposure (Successive) Condition } \\
\hline 4 & 8 & 0 & 4 & 8 & 6 & 6.8 \\
\hline 8 & 4 & 0 & 8 & 4 & 6 & 5.5 \\
\hline 4 & 8 & 4 & 8 & 12 & 10 & 10.2 \\
\hline 8 & 4 & 4 & 12 & 8 & 10 & 9.9 \\
\hline
\end{tabular}


does not demand that spatial disparities at onset provide the depth information. Such an interpretation would require that simultaneously onset exposures yield no illusion, but as shown in the next two rows of Table 1 , illusions are observed under conditions of simultaneous onset and unequal exposure duration. We must conclude, therefore, that while an onset to onset rule predicts the illusion when exposures are equal, it is not the case that the informational basis for the MachDvorak illusion is provided by spatial disparities at target onset. The pairing model that best captures the observed latencies for synchronously onset but unequal exposure appears to be that which assumes that the complete traverses (or, perhaps, "average" positions) are the bases for the illusory displacement.

The last four rows of Table 1, those for the unequal and successive exposures, reiterate these claims; the observed illusions correspond to those predicted by the disparities of the two segments, one to each eye, that arise as the target is successively exposed. Reference to Figure 1 should make this statement clear. If the two entire traverses are, in effect, overlaid one upon the other, position of the target would be on the diagonal connecting the onset-onset intersection to the offsetoffset intersection. Observers, in turn, position the marker somewhere along the diagonal. Indeed, both observers noted such diagonal placement and found that locating the marker was more difficult: The target was seen as intersecting the frontoparallel plane defined by the marker.

Because the traverse pairing algorithm most accurately describes both the equal and unequal exposure duration conditions, it is, of course, the more general rule. Such a rule claims that binocular stereopsis in the Mach-Dvorak situation cannot be described as mapping, fusing, or comparing single points. Rather, the entire visible traverses appear to be the entities over which the disparity is defined. This observation has two implications apart from describing the temporal characteristics of depth information yielded by brief, successive inputs from a moving object. The first bears on results reported by Lee (1970), and the other concerns the use of the illusion as a research tool. Let us consider these two implications in turn.

Lee (1970) investigated the temporal parameters governing the pairing of alternating exposures. In our experiments we had one pair (e.g., right then left) in much closer temporal proximity than the other pair (in this case, left to right). Lee's experiments, on the other hand, had the two intervals much more nearly equal. He then determined which pairing (left-right or right-left) dominated by seeing whether the target appeared to shift toward or away from the observer. Using this dependent measure and unequal exposures, Lee (1970, Experiments 3.1 and 3.2) found that the fusion was of the pair whose offset to onset interval was shorter. At issue between Lee's experiment and ours is whether one set of principles describes the pairing of exposures while another set describes the amount of displacement given a particular pairing. In our earlier paper, we concluded that an offset-onset law held for the former and an onset-onset law held for the latter. As it turns out, the entire traverses rule holds in both cases.

We have already shown how the onset-onset rule gives way to the more general rule. Let us now demonstrate how Lee's (1970) offset-onset rule yields to the total-traverses rule, too. If, for example, a 20-msec exposure is sandwiched between two 10-msec exposures with two intervals of $10 \mathrm{msec}$ (i.e., $10_{\mathrm{L}}-10-20_{\mathrm{R}}-10-10_{\mathrm{L}}$ ), the pairing, by Lee's observation and rule, is equally likely to be left-right or right-left. Moving the 20 -msec exposure toward either $10-\mathrm{msec}$ exposure increases the likelihood that it will be paired with that exposure. To be sure, this represents an offset to onset rule, but note that the manipulation also succeeds in bringing the "total traverses" closer together. Thus, the pairing algorithm can be understood to be a function of which two total traverses are closer together. The offsetonset interpretation is less appealing than the "fusion" of traverses interpretation because the absolute size of the offset-onset interval is quite irrelevant to the size of the illusion (cf. Michaels et al., 1977, Experiment 1 and rows 1.4 of Table 1 in this paper).

In sum, we think that the concept of overlaying two total traverses reconciles the disparities between Lee's report and our own. In Lee's experiment, decreasing offset-onset time brought the two exposed segments closer together in time and space, as did decreasing onsetonset time in our earlier experiment. That the pairing of traverses can disguise itself as two very different relations is owing to our shared and erroneous assumption that single points provide the reference for the Mach-Dvorak illusion.

The second implication of our results is of more general import. Many hold the view that there are two distinct modes of binocular vision, static and kinetic. Moreover, it might be assumed that the Mach-Dvorak illusion, because it is binocular and things are moving, is a token of the latter kind (Lee, 1970). However, it seems to be the case that the illusion can be predicted simply in terms of the "fusion" of two line segments of different lengths. Such fusion yields a single segment that diagonally intersects the frontoparallel plane. This suggests that the Mach-Dvorak effect is not a truly binocular kinetic phenomenon. That is, the observed illusions are precisely those that would be expected from a geometrical analysis of a stationary rod positioned such that its length subtended different visual angles on the two retinae. In the Mach-Dvorak situation, it is simply the case that the lengths of the segments and their relative positions are functions of exposure duration and delay. The implication of these conjectures is that the Mach-Dvorak effect may be a wholly different 
species of illusion from the Pulfrich effect with which it has been closely associated.

At issue is whether the Pulfrich effect is truly binocular kinetic or whether it too can be understood simply with reference to the geometry of a static situation. Recent evidence suggests that it cannot; Morgan and Thompson (1975), Ross (1974), and Wist, Brandt, Diener, and Dichgans (1977) have all mustered convincing arguments that the crucial variables affecting the Pulfrich illusion are temporal, not spatial. We might speculate from this that, while the Mach-Dvorak phenomenon can be used to investigate binocular static vision, albeit inconveniently, it, unlike the Pulfrich phenomenon, is not appropriate for the study of binocular kinetic vision.

In summary, the onset to onset rule of binocular integration in the Mach-Dvorak illusion was investigated. It was found that this rule is limited to conditions of equal exposure to the two eyes. With unequal exposures, either synchronized or asynchronized, a more general rule emerged. This rule claimed that total traverses are functionally fused. The idea of fusing total traverses accounts for both the onset-onset rule and the offsetonset rule that have been proposed in the past to describe the temporal parameters of fusion. The new rule called into question the Mach-Dvorak stereoillusion as a tool appropriate for the study of binocular kinetic vision.

\section{REFERENCE NOTE}

1. Michaels. C. F. The information for direct binocular stereopsis. Manuscript submitted for publication. 1978.

\section{REFERENCES}

LEE, D. N. Spatio-temporal integration in binocular-kinetic space perception. Vision Research, 1970, 10, 65-78.

Michaels. C. F.. Carello, C., Shapiro. B., \& Steitz. C. An onset to onset rule for binocular integration in the Mach-Dvorak illusion. Vision Research, 1977, 17, 1107-1113.

Morgan. M. J., \& Thompson, P. Apparent motion and the Pulfrich effect. Perception, 1975, 4, 3-18.

Ross. J. Stereopsis by binocular delay. Nature, 1974, 248, 363-364.

Wist, E. R.. Brandt, T., Diener, H.-C., \& Dichgans, J. Spatial frequency effect on the Pulfrich stereophenomenon. Vision Research, 1977, 17, 391-397.

\section{NOTES}

1. The present paper and the one that preceded it couch the discussion of binocular vision in terms of the fusion of disparate retinal images. This was done as a matter of convenience and in spite of our conviction that the concept of fusion or integration is in serious need of reformulation (Michaels, Note 1).

2. Unless the observer's head is appropriately constrained by a narrow slit or bite bar, head tilt induces a discrepancy between intended and actual temporal values. This problem may arise with any apparatus in which the functional shut ter acts vertically.

(Received for publication February 23, 1979.) 